\section{Surprising CRISPR roadblocks}

Cas9 induces larger-than-anticipated mutations in mouse and human cells. In the latter, efficient editing depends on inhibition of the DNA-damagerepair protein $\mathrm{p} 53$.

$t$ is an illustration of science at its best:

Michael Kosicki, a PhD student in

Allan Bradley's lab at the Sanger Institute, was using CRISPR to target exons in the $\mathrm{X}$-linked PigA gene and explore the effect of PigA knockout in mouse embryonic stem cells (mESCs). As a control, he targeted an intron in the same gene, expecting any mutations to be spliced out in the mature transcript and thus not lead to any phenotype. Instead, he saw what Bradley describes as "unusual results."

Putting the original project on hold, Kosicki, together with Kärt Tomberg, a postdoctoral fellow working with Bradley, systematically characterized mutations resulting from a double-strand cleavage event introduced by Cas9 (Kosicki et al. 2018).

They compared single guide RNAs (sgRNAs) targeting Cas9 to either intronic or exonic sites in PigA in mESCs from male mice. At first sight all seemed well, and a majority of cells targeted at the exonic site showed loss of PigA. Surprisingly, though, up to $20 \%$ of cells targeted at the intronic site also lacked PigA, mostly due to large deletions in the kilobase range that overlapped the adjacent exons on either side of the cut site. When the team isolated single-cell clones with an edited PigA locus, they saw that $17 \%$ of sequenced alleles harbored additional lesions away from the cut site, including inversions and insertions; in some instances the inserted sequences mapped to unrelated parts of the mouse genome.

Intrigued by these results, Bradley and his team wondered whether these findings were specific to the monoallelic X-linked PigA in male mESCs. They next targeted the autosomal $C d 9$ gene, which encodes a cell surface protein that is not essential for mESCs. The results were similar: single-cellderived edited clones predominantly showed large deletions.

"We have been lulled into the view that editing is small and local and controllable," says Bradley, "but the reality of DNA repair in a cell is much more complex."

Two other recent studies underscore the complexity of the Cas9-induced repair process in cells. Teams led by Bernhard Schmierer and Jussi Taipale at the Karolinska Institute showed that in human retinal pigment epithelial cells, intact tumor suppressor TP53 triggers cell-cycle arrest and drastically reduces the efficiency of gene editing (Haapaniemi et al. 2018). Short-term inhibition of TP53 increases the editing rate but renders the cells more vulnerable to chromosomal rearrangements and mutations that could be tumorigenic. Work directed by Ajamete Kaykas at the Novartis Institute for Biomedical Research highlighted similar inefficient Cas9-based editing in human ESCs (Ihry et al. 2018). The researchers discovered a p53-dependent toxic response to DNA double-strand breaks introduced by single sgRNA-Cas 9 complexes. This toxicity could lead to a selection of cells with p53 defects that better tolerate DNA damage.

In animal experiments, where genetic lesions can be eliminated through breeding, unwanted mutations are relatively easy to remove. But when CRISPR-edited cells are used therapeutically to correct a somatic defect, there could be more harm, as even a few cells with a mutation that confers a growth advantage could lead to a bad outcome for a patient.

More work is needed to fully understand the range and cause of undesired editing outcomes. In the meantime, it is important to catalog the full scope of Cas9's action to see whether it is as precise and innocuous as many expect it to be.

"Look carefully" is Bradley's take-home message.

Nicole Rusk

Published online: 31 July 2018 https://doi.org/10.1038/s41592-018-0097-9

\section{Research papers}

Kosicki, M. et al. Repair of double-strand breaks induced by CRISPR-Cas9 leads to large deletions and complex rearrangements. Nat. Biotechnol. https://doi. org/10.1038/nbt.4192 (2018).

Haapaniemi, E. et al. CRISPR-Cas9 genome editing induces a p53-mediated DNA damage response. Nat. Med. 24, 927-930 (2018).

Ihry, R. J. et al. p53 inhibits CRISPR-Cas9 engineering in human pluripotent stem cells. Nat. Med. 24, 939-946 (2018).

\section{Worthington}

The Feeling is Mutual

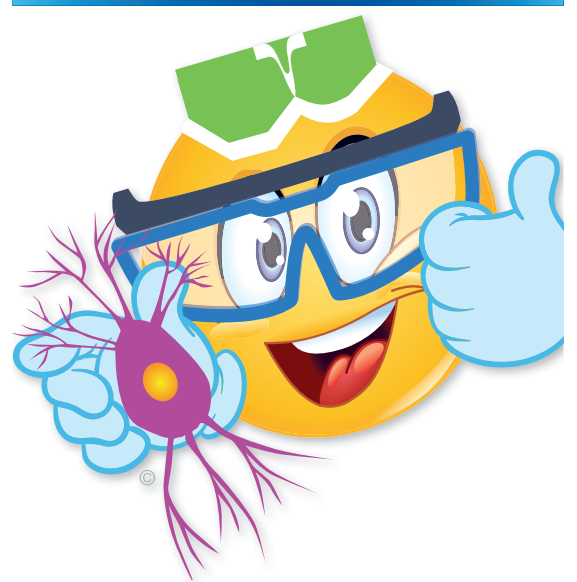

We love our customers and it seems they love us too!

\author{
With over 100 citations \\ published in leading scientific \\ journals monthly, Worthington \\ enzymes have been critical to \\ research for 70 years.
}

\section{Authoring technical manuals and guides both print and digital, we look to serve the needs of up-and-coming research scientists worldwide.}

\section{Feeling it's time to discover Worthington enzymes? Visit: Worthington-Biochem.com}
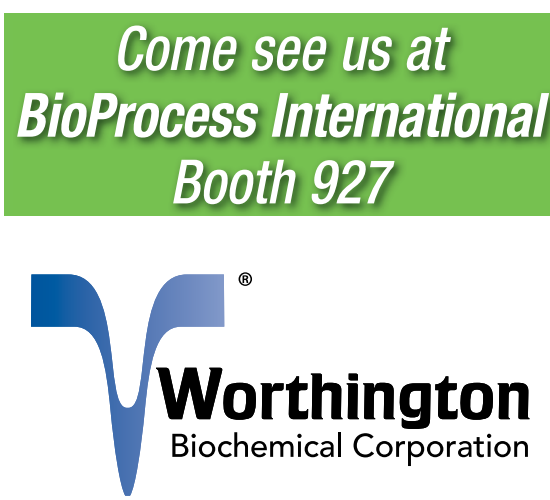

$800.445 .9603 \bullet 732.942 .1660$ 